OPEN ACCESS

Edited by:

Harrison Bai,

Brown University, United States

Reviewed by:

Jiaojian Wang

University of Electronic Science and

Technology of China, China

Wei Wei,

Xi'an Polytechnic University, China

*Correspondence:

Songbai Gui

guisongbai@yeah.net

Specialty section:

This article was submitted to

Cancer Imaging and Image-directed Interventions,

a section of the journal

Frontiers in Oncology

Received: 28 August 2020 Accepted: 30 December 2020 Published: 17 February 2021

Citation:

Ma G, Kang J, Qiao N, Zhang B, Chen X, Li G, Gao Z and Gui S (2021) Non-Invasive Radiomics Approach Predict Invasiveness of Adamantinomatous Craniopharyngioma Before Surgery.

Front. Oncol. 10:599888.

doi: 10.3389/fonc.2020.599888

\section{Non-Invasive Radiomics Approach Predict Invasiveness of Adamantinomatous Craniopharyngioma Before Surgery}

\author{
Guofo Ma ${ }^{1}$, Jie Kang ${ }^{1}$, Ning Qiao ${ }^{1}$, Bochao Zhang ${ }^{1}$, Xuzhu Chen ${ }^{2}$, Guilin $L^{3}$, \\ Zhixian $\mathrm{Gao}^{1}$ and Songbai Gui ${ }^{{ }^{*}}$
}

1 Department of Neurosurgery, Beijing Tiantan Hospital, Capital Medical University, Beijing, China, ${ }^{2}$ Department of Radiology, Beijing Tiantan Hospital, Capital Medical University, Beijing, China, ${ }^{3}$ Neuropathology Department, Beijing Neurosurgical Institute, Capital Medical University, Beijing, China

Purpose: Craniopharyngiomas (CPS) are benign tumors, complete tumor resection is considered to be the optimal treatment. However, although histologically benign, the local invasiveness of CPs commonly contributes to incomplete resection and a poor prognosis. At present, some advocate less aggressive surgery combined with radiotherapy as a more reasonable and effective means of protecting hypothalamus function and preventing recurrence in patients with tight tumor adhesion to the hypothalamus. Hence, if a method can be developed to predict the invasiveness of CP preoperatively, it will help in the development of a more personalized surgical strategy. The aim of the study was to report a radiomics-clinical nomogram for the individualized preoperative prediction of the invasiveness of adamantinomatous CP (ACPs) before surgery.

Methods: In total, 1,874 radiomics features were extracted from whole tumors on contrast-enhanced T1-weighted images. A support vector machine trained a predictive model that was validated using receiver operating characteristic $(\mathrm{ROC})$ analysis on an independent test set. Moreover, a nomogram was constructed incorporating clinical characteristics and the radiomics signature for individual prediction.

Results: Eleven features associated with the invasiveness of ACPs were selected by using the least absolute shrinkage and selection operator (LASSO) method. These features yielded area under the curve (AUC) values of 79.09 and $73.5 \%$ for the training and test sets, respectively. The nomogram incorporating peritumoral edema and the radiomics signature yielded good calibration in the training and test sets with the AUCs of 84.79 and $76.48 \%$, respectively.

Conclusion: The developed model yields good performance, indicating that the invasiveness of APCs can be predicted using noninvasive radiological data. This reliable, noninvasive tool can help clinical decision making and improve patient prognosis.

Keywords: craniopharyngioma, adamantinomatous, invasiveness, radiomics, machine learning, nomogram 


\section{INTRODUCTION}

Craniopharyngiomas (CPs) are rare and non-neuroepithelial entities arising from a malformation of embryonal tissue, with an incidence of $0.5-2$ cases per million persons per year (1-3). Two histological subtypes have been identified: adamantinomatous CPs (ACPs) and papillary CPs (PCPs). They are commonly located in the suprasellar region and can cause devastating neuroendocrine dysfunction by mass effect and/or invasion to the optic apparatus, pituitary gland and hypothalamus. Complete tumor resection with improvement in visual function, and no further deterioration of neuroendocrine and cognitive function is considered the optimal treatment outcome. However, although these massed are of a benign histological nature, the abovementioned ideal treatment goal is not always achievable due to the potential close adhesion of CPs to surrounding brain tissue.

Pathological studies have confirmed that the histology of the interface between CPs and surrounding brain tissue can be classified into two types, including finger-like invasion and no finger-like invasion (4-6). Numerous investigators have deemed that such local invasion resulting in adhesion could be associated with the failure of complete resection and poor prognosis (7-9). Therefore, a preoperative noninvasive method for identifying the invasiveness of CPs could help in the development of more individualized treatment decisions. Addressing this problem, we developed a machine learning radiomics model to predict the invasiveness of ACPs before surgery.

Radiomics is an emerging research method that can effectively evaluate the heterogeneity of tumors by extracting a large number of image features from medical images. Its applicability and utility have already been validated in several tumor types; Zhang et al. focused on the preoperative prediction of nonfunctioning pituitary adenoma subtypes before surgery (10); Li et al. predicted P53 status, progression-free survival (PFS), phosphatase and tensin homolog (PTEN) and vascular endothelial growth factor (VEGF) expression in patients with gliomas (11-14). Furthermore, radiomics approaches have also been validated in meningiomas (15), lung cancer (16) and skull base chordomas (17).

In the current study, we extracted a large number of radiomics features from preoperative MRI scans of ACPs with known local invasiveness. We hypothesized that a radiomics model could predict the invasiveness of ACPs via a machinelearning algorithm.

\section{METHODS}

\section{Patients}

We retrospectively reviewed the medical records of patients who underwent surgery for craniopharyngioma from 2002 to 2019, and a total 335 cases of ACPs were included in this study. Their radiographic and pathological data were collected from picture archiving and communications systems. The pathological sections were reviewed by two individual senior neuropathologists to confirm the histology of the interface between the ACPs and surrounding brain tissue (Figures S2 and S4). Potential candidates were excluded if their pathological sections could not reflect the relationship between the ACP and brain tissues. Furthermore, MRI images were reviewed by two experienced radiologists to identify whether peritumor edema was present on T2-weighted images. Any disagreement was resolved by a consultation. The inclusion criteria were as follows: 1) histologically confirmed as ACPs; 2) the definite invasiveness of each tumor; 3) complete preoperative MRI data [including T2-weighted, T1-weighted and contrast enhanced (CE)-T1 images]; 4) no history of surgical treatment; and 5) available clinical characteristics. Among 335 patients, 225 patients who were treated between January 2002 and December 2015 were allocated to the training set, and 110 patients who were treated between January 2016 and December 2019 were allocated to the validating set. The training set was used to establish a stable model to predict the invasiveness of ACPs via radiomics features, while the validation set was used to assess the prediction accuracy of the model. The study was approved and reviewed by the institutional review board.

\section{MRI Acquisition and Tumor Segmentation}

CE-T1 images were used for the extraction of radiomics features, as these images are optimal for identifying the tumor border. MRI was performed in the head-first supine position on a $3-T$ scanner (Tim Trio, Siemens) using a head coil. The acquisition parameters for precontrast T1-weighted sequences were as follows: repetition time, $156-2,520 \mathrm{~ms}$; echo time, $2-19.7 \mathrm{~ms}$; flip angle: $150^{\circ}$; field of view: $240 \times 188 \mathrm{~mm}^{2}$; acquisition matrix: $384 \times 300$ and slice thickness: $5 \mathrm{~mm}$. The study was repeated immediately after the rapid injection of contrast agent gadolinium-DTPA $(0.1 \mathrm{mmol} / \mathrm{kg}$ Gadovist; Beijing Beilu Pharmaceutical Co., Beijing China). The regions of interest (ROI), i.e., whole tumors, were manually delineated by two neuroradiologists on the CE-T1 images using MRIcron software (http://www.mccauslandcenter.sc.edu/mricro) (Figure S1 and S3). The two neuroradiologists were blinded to the patients' clinical characteristics. Next, a third senior neuroradiologist reevaluated the ROIs and made final decisions when discrepancies were $\geq 5 \%$.

\section{Feature Extraction}

First, we homogenized the image intensity on all MR images by z-score transformation (MATLAB version 2014a; The Mathworks, Natick, MA, USA) to avoid heterogeneity bias. In this study, a total of 1,874 features were acquired (Table S1). The features were divided into eight categories: (a) first-order statistics, (b) shape-based, (c) Gray Level Cooccurence Matrix (GLCM), (d) Gray Level Run Length Matrix (GLRLM), (e) Gray Level Size Zone Matrix (GLSZM), (f) Neighboring Gray Tone Difference Matrix (NGTDM), (g) Gray Level Dependence Matrix (GLDM), and (h) wavelet features, they were derived from firstorder statistics and texture features via wavelet decomposition (using directional low-pass and high-pass filtering.

\section{Feature Selection and Classification}

We used the least absolute shrinkage and selection operator (LASSO) algorithm, which is a suitable and powerful method for 
the regression of high-dimensional data, to screen the most predictive features in the training set. In this procedure, the tuning parameter (lambda) was selected by the cross-validation method; the optimal lambda was confirmed as that which resulted in the smallest cross-validation error. Then, a support vector machine (SVM) classifier was used to establish a machine-learning model for invasiveness prediction. The performance of the classification model was evaluated and validated by employing 10-fold cross-validation. Receiver operator characteristic (ROC) curve analysis was performed for both the training and validation sets to evaluate the discriminative ability of the machine-learning model.

\section{Radiomics-Clinical Nomogram Construction and Performance Assessment}

To provide a more individualized predictive model, a nomogram was built from the training set data. First, a radiomics signature was constructed using the selected features, and represented by a radiomics score. The score was calculated for each patient as a linear combination of the selected features weighted by their respective coefficients. Second, the radiomics signature and other clinical predictors (age, sex, peritumoral edema, tumor size) were tested using a multivariate logistic regression algorithm in the training set. The final selection of the model for the nomogram

TABLE 1 | Patient characteristics.

\begin{tabular}{lccc}
\hline & Training & Validation & P value \\
\hline Age(years, mean) & 37.2 & 35.63 & $0.61^{\mathrm{a}}$ \\
Sex(Male/Female) & $129 / 96$ & $58 / 52$ & $0.43^{\mathrm{b}}$ \\
Peritumoral edema & $48 / 177$ & $19 / 91$ & $0.38^{\mathrm{b}}$ \\
Tumor invasiveness & $65 / 120$ & $31 / 79$ & $0.22^{\mathrm{b}}$ \\
\hline
\end{tabular}

${ }^{a}$ Mann-Whitney $U$ test, ${ }^{b}$ Chi-square test. was conducted using a backward step-down selection process based on the Akaike information criterion. The performance of the nomogram was estimated with the training cohort and then tested with the validation cohort.

\section{Statistics}

The Mann-Whitney $U$ test and chi-square test were used to evaluate whether age, sex, tumor invasiveness and peritumoral edema were significantly different between the training set and validation set. They were performed by using SPSS software version 22.0 (IBM Corp.) Statistical significance was set as $\mathrm{p}<$ 0.05 . The LASSO algorithm, SVM classifier, ROC curve analysis and nomogram were performed based on "glmnet", "e1071", "pROC", and "rms" packages in R software version 3.3.2 (The R Foundation, Salt Lake City, UT, USA), respectively.

\section{RESULTS}

\section{Clinical Characteristics}

A total 187 male and 148 female patients was enrolled in the study, with 51 pediatric patients (mean age 14.3 years, range 617 years) and 284 adult patients (mean age 41.6 years, range 18 71 years). Among these patients, 129 men and 96 women were allocated to the training group and 58 men and 52 women were allocated to the validation group via random assignment. The ratios of invasiveness to noninvasiveness were $65 / 160$ in the training group and $31 / 79$ in the validation set. The distributions of the characteristics of the two groups were compared using the Mann-Whitney U test and the chi-square test, and there were no significant differences in age $(p=0.61)$, sex $(p=0.43)$, peritumoral edema $(\mathrm{p}=0.38)$, tumor size or invasiveness $(\mathrm{p}=$ 0.22). Detailed information pertaining to the clinical characteristics of the patients is shown in Table $\mathbf{1}$.
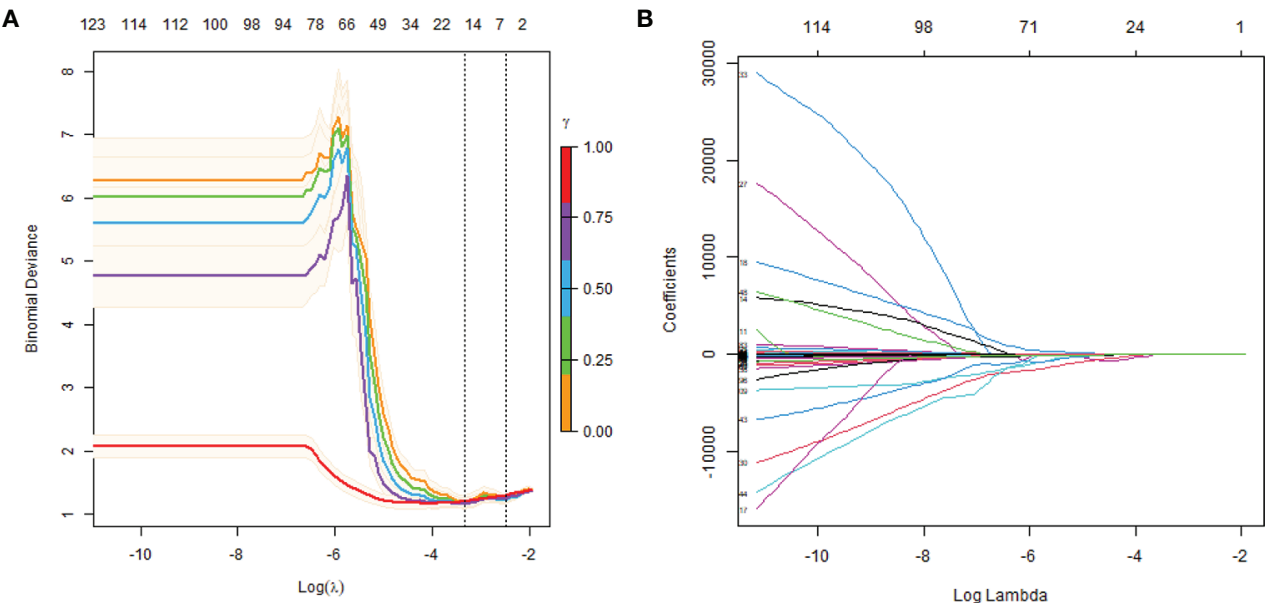

FIGURE 1 | Texture feature selection using LASSO logistic regression. (A) Selection of the tuning parameter (lambda). The dotted vertical lines are plotted at the optimal $\lambda$ values based on the minimum criteria and 1 standard error of the minimum criteria. (B) LASSO coefficient profiles are shown for the 1874 texture features. A vertical line is drawn at the value where the optimal lambda results in 11 nonzero coefficients. 


\section{Machine-Learning Model for Predicting the Invasiveness of ACPs}

In this study, the LASSO algorithm was used to select features with nonzero coefficients, and a subset of 11 features were screened from a total of 1,874 radiomic features (Figures 1A, B). The names and descriptions of these 11 selected features are shown in Table 2.

A machine-learning model was constructed based on the selected features and the SVM classifier with the training set data. The AUC was $79.09 \%$ following ROC curve analysis, and the sensitivity, specificity, and accuracy were $81.97 \%, 66.74$ and $75 \%$, respectively at the optimal cutoff point (0.609) (Figure 2A). Then, the model was applied to the validation set, and the invasiveness of the ACPs was effectively predicted. In the ROC curve analysis, the AUC was $73.5 \%$. In addition, the optimal cutoff value (0.568) yielded a sensitivity, specificity, and accuracy of $69.53,72.44$, and $66.53 \%$, respectively (Figure 2B). Hence, the 11 radiological features that constituted our model were regarded as an effective radiomics signature for the invasiveness of ACPs.

\section{Development and Validation of the Individualized Predictive Nomogram}

The radiomics signature and peritumoral edema were identified as independent predictors of ACP invasiveness based on the multivariate logistic regression algorithm (Table 3). The nomogram showed favorable discrimination with an AUC of 84.79\% [95\% confidence interval (CI), 84.12-85.46\%] in the training set (Figures 3A, B). The radiomic nomogram also showed good discrimination with an AUC of $76.48 \%$ (95\% CI, 74.13-78.83\%) in the testing set (Figure 3C).

\section{DISCUSSION}

Although the history of surgical treatment for CPs has been spanned the course of more than 100 years, these masses still pose a surgical challenge even after the application of modern neurosurgical techniques (18-22). Numerous studies have revealed that quality of life (QoL) and cognitive performance

TABLE 2 | Eleven prognostic radiomics features selected by the LASSO algorithm.

Features

Descriptions

Coefficients

First order_Skewness

GLSZM_ Gray Level Variance

Shape_Sphericity

Shape _ Surface Volume Ratio

GLCM _ Contrast

wavelet-HLL_GLDM_DNU

wavelet-LHL_GLCM_

Autocorrelation

wavelet-HLH_NGTDM_Busyness

wavelet-LLL_NGTDM _Complexity wavelet-HLL_ GLSZM_SAHGLE wavelet-LLH_GLSZM_SZNUN
Skewness measures the asymmetry of the distribution of values about the Mean value.

Measuring the variance in gray level intensities for the zones.

Measuring the roundness of the shape of the tumor region relative to a circle

A lower value indicates a more compact (sphere-like) shape and dependent on the volume of the ROI.

Measuring the local intensity variation, favoring values away from the diagonal.

Describing the homogeneity among dependencies in the image. The value is low if the image has more similarity.

Describing the magnitude of the fineness and coarseness of texture.

Describing the change from a pixel to its neighbor. The value is high if the changes of intensity between pixels and its neighborhood is rapid.

Describing the complexity of the image. The value is high if there are many rapid changes in gray level intensity.

Describing the distribution of smaller size zones with higher gray-level values.

Describing the variability of size zone volumes throughout the image.
$-2.7 \times 10^{-1}$

$4.32 \times 10^{-1}$

$1.13 \times 10^{-1}$

$2.46 \times 10^{-2}$

$2.87 \times 10^{-3}$

$-5.1 \times 10^{-1}$

$-6.72 \times 10^{-2}$

$-1.91$

$4.09 \times 10^{-3}$

$-1.17 \times 10^{-1}$

$-7.42 \times 10^{-2}$

DNU, Dependence Non Uniformity; SAHGLE, Small Area High Gray Level Emphasis; SZNUN, Size Zone Non Uniformity Normalized.

A

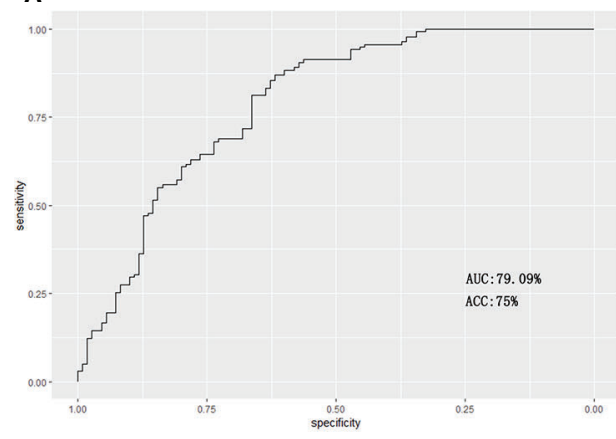

B

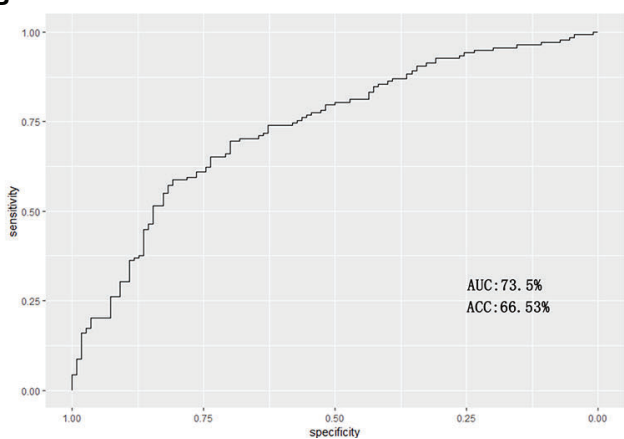

FIGURE 2 | Receiver operating characteristic curves for the prediction of invasiveness of ACPs in the training and validation sets. (A) For the training set, the area under the curve (AUC) was 79.09\% with a sensitivity, specificity and accuracy of $81.97,66.74$, and $75 \%$, respectively. (B) For the validation set, the AUC was $73.5 \%$ with a sensitivity, specificity and accuracy of $69.53,72.44$, and $66.53 \%$, respectively. 
TABLE 3 | Multivariate logistic regression analysis of the radiomics score and clinical predictors in the training set.

\begin{tabular}{|c|c|c|c|c|c|c|}
\hline & \multicolumn{3}{|c|}{ Univariate logistic regression } & \multicolumn{3}{|c|}{ Multivariate logistic regression } \\
\hline & HR & $95 \% \mathrm{Cl}$ & $P$ value & HR & $95 \% \mathrm{Cl}$ & $P$ value \\
\hline Age, per 1 year increase & 0.927 & $0.552-1.556$ & 0.77 & & & \\
\hline Sex (male) & 0.988 & $0.972-1.005$ & 0.16 & & & \\
\hline Peritumoral edema & 2.499 & $1.351-4.624$ & 0.014 & 1.964 & $1.018-3.788$ & 0.036 \\
\hline Tumor size, per $1 \mathrm{~cm}$ increase & 1.719 & $1.899-3.288$ & 0.101 & & & \\
\hline Radiomics score, per 0.1 increase & 1.583 & $1.079-2.458$ & $<0.001$ & 1.257 & $1.072-1.473$ & 0.005 \\
\hline
\end{tabular}

A

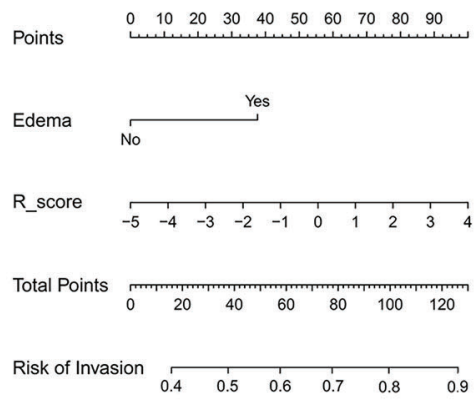

B

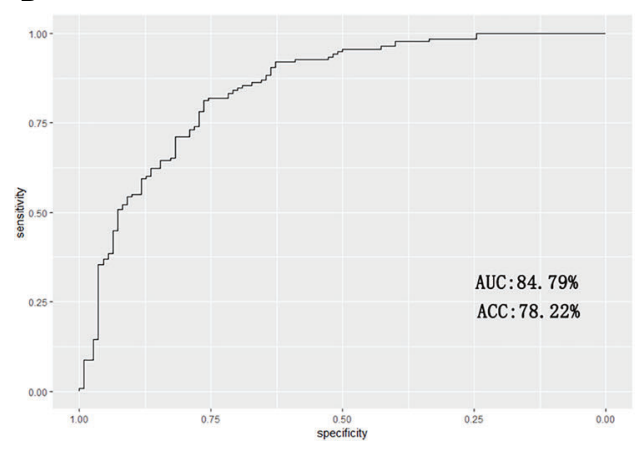

C

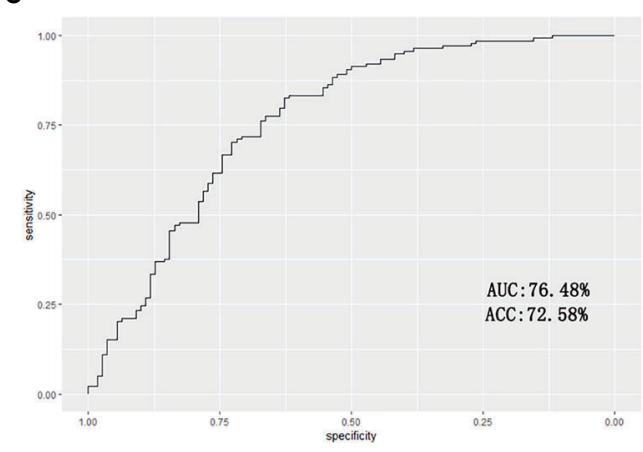

FIGURE 3 | The radiomic-clinical nomogram and its performance are illustrated. (A) The radiomics-clinical nomogram developed to predict the invasiveness of ACPs is illustrated. (B) For the training set, the AUC was $84.79 \%$ with the sensitivity, specificity and accuracy of $83.27,76.05$, and $78.22 \%$, respectively. (C) For the validation set, the AUC was $76.48 \%$ with a sensitivity, specificity and accuracy of $71.24,72.33$, and $72.58 \%$, respectively.

are frequently impaired in long-term survivors after surgery due to the anatomical proximity of the CPs to the optic nerve and to the hypothalamic-pituitary axes (23-25). Some researchers have advocated less aggressive surgery combined with radiotherapy as a more reasonable and effective means of protecting hypothalamus function and preventing recurrence in the patients with tight tumor adhesion to the hypothalamus (2628). Therefore, it is important to assess the aggressiveness of the tumor before surgery. In the present study, we used the noninvasive radiomics method to predict the invasiveness of ACPs before surgery, which made it possible to develop a personalized surgical protocol. Note that to avoid heterogeneity between the two histopathological CP subtypes, only ACPs were included in the study.

In our cohort, there were more male patients $(\mathrm{n}=187$, $55.82 \%)$ than female patients $(\mathrm{n}=148,44.18 \%)$, consistent with previous reports (29-31). Although CPs were more common among child patients, the proportion of adult patients was higher in this study ( 84.78 vs. $15.22 \%)$; because adult patients were the main group of patients in our ward.

To date, radiomics studies on craniopharyngiomas are rare. Yue et al. proposed a machine learning model for discriminating BRAF mutation and wild type among craniopharyngiomas with sensitivity of 1.00 and specificity of 0.91 (32). Chen et al. predicted the pathological subtype and gene mutations in craniopharyngiomas with radiomics (33).

Radiomics is an emerging diagnostic technique, and the potential ability of improving clinical decision support systems has been well verified. Some successful precedents have been demonstrated in radiomics studies for identifying the invasiveness of tumors. For example, a previous report showed that preinvasive pulmonary adenocarcinomas and invasive pulmonary adenocarcinomas could be distinguished by constructing a radiomics-clinical nomogram predictive model 
with an AUC of 0.903 (34). Another report revealed that the muscular invasiveness of bladder cancer could be evaluated by a noninvasive radiomics model (35). Furthermore, Zhu et al. proposed a learning radiomics model for preoperative grading in meningioma (36). In the present study, we employed a radiomics approach to provide preoperatively predict the invasiveness of ACPs. The high-throughput features applied in our radiomics model were extracted from the whole tumor on preoperative CE-T1 images, which could reflect the heterogeneity of the tumor. Subsequently, 11 invasiveness-associated features were screened by using the LASSO algorithm, consisting of one first-order feature, two shape-based features, two texture features, and six wavelet features. Most of these selected features were also reported in previous studies of tumor invasiveness $(35,37)$. Our predicted model constructed by using an SVM classifier achieved AUCs of $79.09 \%$ in the training set data and $73.5 \%$ in the validation set data. The results indicate that the invasiveness of APCs can be predicted using noninvasive radiological data, and the proposed radiomics signature performed well in the training and validation sets.

Tumor invasiveness is closely associated with gene mutations and/or relative protein expression levels. However, owing to its rarity and benign histological nature, studies of the genomics and molecular pathology of CPs are limited. A previous study revealed that the expression of claudin-1, a tight junction protein expressed in epithelial tissues that plays important roles in cell polarity and adhesion, could be strongly associated with the invasiveness of CPs (38). The authors found that the invasive CPs exhibited significantly lower claudin-1 expression than their noninvasive counterparts regardless of $\mathrm{CP}$ subtype. We suggest that this difference may be the basis of the molecular pathology for distinguishing invasive and noninvasive ACPs by using the radiomics method.

The individualized predictive nomogram, incorporated the radiomics signature and peritumoral edema into a model, which facilitated the individualized prediction of the invasiveness of ACPs. The radiomic-clinical nomogram showed better discrimination in the training and validation sets with AUCs of 84.79 and $76.48 \%$, respectively. This revealed that combining multiple clinical risk factors to estimate and determine follow-up treatment, rather than focusing on a single radiological feature, is very necessary.

There are some limitations in our study. First, to build the radiomics signature and predictive model, we analyzed axial CET1 images, which are usually referred to clinically; however, combinations with other sequences such as fluid attenuated inversion recovery (FLAIR) imaging and T2-weighted imaging may have provided additional information and improved the

\section{REFERENCES}

1. Garrè ML, Cama A. Craniopharyngioma: modern concepts in pathogenesis and treatment. Curr Opin Pediatr (2007) 19(4):471-9. doi: 10.1097/ MOP.0b013e3282495a22

2. Nielsen EH, Feldt-Rasmussen U, Poulsgaard L, Kristensen LO, Astrup J, Jørgensen JO, et al. Incidence of craniopharyngioma in Denmark ( $\mathrm{n}=189$ ) and estimated world incidence of craniopharyngioma in children and adults. J Neuro-Oncol (2011) 104(3):755-63. doi: 10.1007/s11060-011-0540-6 performance of the predictive model. Second, potential selection biases might have occurred because of the retrospective nature of the study. Third, the imaging protocols used were not fully consistent in that the imaging data were acquired with different MRI scanners.

\section{CONCLUSION}

We proposed a radiomics-clinical nomogram for the individualized preoperative prediction of the invasiveness of ACPs. This reliable, noninvasive tool can help clinical decision making and improve patient prognosis.

\section{DATA AVAILABILITY STATEMENT}

The raw data supporting the conclusions of this article will be made available by the authors, without undue reservation.

\section{ETHICS STATEMENT}

The studies involving human participants were reviewed and approved by The Ethics Committee of Beijing Tiantan Hospital affiliated to Capital Medical University. Written informed consent to participate in this study was provided by the participants' legal guardian/next of kin.

\section{AUTHOR CONTRIBUTIONS}

GM collected and analyzed clinical data, and prepared the manuscript. SG designed the trial and revised the manuscript. NQ and BZ collected clinical data. XC supervised the tumor segmentation. GL supervised the evaluation of the histology of the interface between CPs and surrounding brain tissue. All authors contributed to the article and approved the submitted version.

\section{SUPPLEMENTARY MATERIAL}

The Supplementary Material for this article can be found online at: https://www.frontiersin.org/articles/10.3389/fonc.2020. 599888/full\#supplementary-material

3. Bunin GR, Surawicz TS, Witman PA, Preston-Martin S, Davis F, Bruner JM. The descriptive epidemiology of craniopharyngioma. J Neurosurg (1998) 89 (4):547-51. doi: 10.3171/jns.1998.89.4.0547

4. Kawamata T, Kubo O, Hori T. Histological findings at the boundary of craniopharyngiomas. Brain Tumor Pathol (2005) 22(2):75-8. doi: 10.1007/ s10014-005-0191-4

5. Liu Y, Qi ST, Wang CH, Pan J, Fan J, Peng JX, et al. Pathological Relationship Between Adamantinomatous Craniopharyngioma and Adjacent Structures Based on QST Classification. J Neuropathol Exp Neurol (2018) 77(11):101723. doi: 10.1093/jnen/nly083 
6. Burghaus S, Hölsken A, Buchfelder M, Fahlbusch R, Riederer BM, Hans V, et al. A tumor-specific cellular environment at the brain invasion border of adamantinomatous craniopharyngiomas. Virchows Archiv an Int J Pathol (2010) 456(3):287-300. doi: 10.1007/s00428-009-0873-0

7. Sterkenburg AS, Hoffmann A, Gebhardt U, Warmuth-Metz M, Daubenbüchel AM, Müller HL. Survival, hypothalamic obesity, and neuropsychological/ psychosocial status after childhood-onset craniopharyngioma: newly reported long-term outcomes. Neuro-oncology (2015) 17(7):1029-38. doi: 10.1093/neuonc/nov044

8. Gautier A, Godbout A, Grosheny C, Tejedor I, Coudert M, Courtillot C, et al. Markers of recurrence and long-term morbidity in craniopharyngioma: a systematic analysis of 171 patients. J Clin Endocrinol Metab (2012) 97 (4):1258-67. doi: 10.1210/jc.2011-2817

9. Fjalldal S, Holmer H, Rylander L, Elfving M, Ekman B, Osterberg K, et al. Hypothalamic involvement predicts cognitive performance and psychosocial health in long-term survivors of childhood craniopharyngioma. J Clin Endocrinol Metab (2013) 98(8):3253-62. doi: 10.1210/jc.2013-2000

10. Zhang S, Song G, Zang Y, Jia J, Wang C, Li C, et al. Non-invasive radiomics approach potentially predicts non-functioning pituitary adenomas subtypes before surgery. Eur Radiol (2018) 28(9):3692-701. doi: 10.1007/s00330-0175180-6

11. Li Y, Qian Z, Xu K, Wang K, Fan X, Li S, et al. MRI features predict p53 status in lower-grade gliomas via a machine-learning approach. NeuroImage Clin (2018) 17:306-11. doi: 10.1016/j.nicl.2017.10.030

12. Liu X, Li Y, Qian Z, Sun Z, Xu K, Wang K, et al. A radiomic signature as a noninvasive predictor of progression-free survival in patients with lower-grade gliomas. NeuroImage Clin (2018) 20:1070-7. doi: 10.1016/j.nicl.2018.10.014

13. Li Y, Liang Y, Sun Z, Xu K, Fan X, Li S, et al. Radiogenomic analysis of PTEN mutation in glioblastoma using preoperative multi-parametric magnetic resonance imaging. Neuroradiology (2019) 61(11):1229-37. doi: 10.1007/ s00234-019-02244-7

14. Sun Z, Li Y, Wang Y, Fan X, Xu K, Wang K, et al. Radiogenomic analysis of vascular endothelial growth factor in patients with diffuse gliomas. Cancer Imaging Off Publ Int Cancer Imaging Soc (2019) 19(1):68. doi: 10.1186/ s40644-019-0256-y

15. Zhang J, Yao K, Liu P, Liu Z, Han T, Zhao Z, et al. A radiomics model for preoperative prediction of brain invasion in meningioma non-invasively based on MRI: A multicentre study. EBioMedicine (2020) 58. doi: 10.1016/ j.ebiom.2020.102933 102933.

16. Shi L, He Y, Yuan Z, Benedict S, Valicenti R, Qiu J, et al. Radiomics for Response and Outcome Assessment for Non-Small Cell Lung Cancer. Technol Cancer Res Treat (2018) 17. doi: 10.1177/1533033818782788 1533033818782788.

17. Wei W, Wang K, Liu Z, Tian K, Wang L, Du J, et al. Radiomic signature: A novel magnetic resonance imaging-based prognostic biomarker in patients with skull base chordoma. Radiother Oncol J Eur Soc Ther Radiol Oncol (2019) 141:239-46. doi: 10.1016/j.radonc.2019.10.002

18. Gardner PA, Prevedello DM, Kassam AB, Snyderman CH, Carrau RL, Mintz $\mathrm{AH}$. The evolution of the endonasal approach for craniopharyngiomas. J Neurosurg (2008) 108(5):1043-7. doi: 10.3171/jns/2008/108/5/1043

19. Yang I, Sughrue ME, Rutkowski MJ, Kaur R, Ivan ME, Aranda D, et al. Craniopharyngioma: a comparison of tumor control with various treatment strategies. Neurosurg Focus (2010) 28(4):E5. doi: 10.3171/2010.1.Focus09307

20. Jeswani S, Nuño M, Wu A, Bonert V, Carmichael JD, Black KL, et al. Comparative analysis of outcomes following craniotomy and expanded endoscopic endonasal transsphenoidal resection of craniopharyngioma and related tumors: a single-institution study. J Neurosurg (2016) 124(3):627-38. doi: 10.3171/2015.3.Jns142254

21. Chakrabarti I, Amar AP, Couldwell W, Weiss MH. Long-term neurological, visual, and endocrine outcomes following transnasal resection of craniopharyngioma. J Neurosurg (2005) 102(4):650-7. doi: 10.3171/ jns.2005.102.4.0650

22. Ozgural O, Kahilogullari G, Dogan I, Al-Beyati ESM, Bozkurt M, Tetik B, et al. Single-Center Surgical Experience of the Treatment of Craniopharyngiomas With Emphasis on the Operative Approach: Endoscopic Endonasal and Open Microscopic Transcranial Approaches. J Craniofac Surg (2018) 29(6):e572-8. doi: $10.1097 /$ scs.0000000000004592
23. Müller HL. Childhood craniopharyngioma-current concepts in diagnosis, therapy and follow-up. Nat Rev Endocrinol (2010) 6(11):609-18. doi: 10.1038/ nrendo.2010.168

24. Müller HL. Childhood craniopharyngioma: current controversies on management in diagnostics, treatment and follow-up. Expert Rev Neurother (2010) 10(4):515-24. doi: 10.1586/ern.10.15

25. Muller HL. Childhood craniopharyngioma. Recent advances in diagnosis, treatment and follow-up. Horm Res (2008) 69(4):193-202. doi: 10.1159/ 000113019

26. Wen BC, Hussey DH, Staples J, Hitchon PW, Jani SK, Vigliotti AP, et al. A comparison of the roles of surgery and radiation therapy in the management of craniopharyngiomas. Int J Radiat Oncol Biol Phys (1989) 16(1):17-24. doi: 10.1016/0360-3016(89)90005-9

27. Hetelekidis S, Barnes PD, Tao ML, Fischer EG, Schneider L, Scott RM, et al. 20-year experience in childhood craniopharyngioma. Int J Radiat Oncol Biol Phys (1993) 27(2):189-95. doi: 10.1016/0360-3016(93)90227-m

28. Rajan B, Ashley S, Gorman C, Jose CC, Horwich A, Bloom HJ, et al. Craniopharyngioma-a long-term results following limited surgery and radiotherapy. Radiother Oncol: J Eur Soc Ther Radiol Oncol (1993) 26(1):110. doi: 10.1016/0167-8140(93)90019-5

29. Feng Y, Ni M, Wang YG, Zhong LY. Comparison of neuroendocrine dysfunction in patients with adamantinomatous and papillary craniopharyngiomas. Exp Ther Med (2019) 17(1):51-6. doi: 10.3892/ etm.2018.6953

30. Goschzik T, Gessi M, Dreschmann V, Gebhardt U, Wang L, Yamaguchi S, et al. Genomic Alterations of Adamantinomatous and Papillary Craniopharyngioma. J Neuropathol Exp Neurol (2017) 76(2):126-34. doi: 10.1093/jnen/nlw116

31. Kiliç M, Can SM, Özdemir B, Tanik C. Management of Craniopharyngioma. J Craniofac Surg (2019) 30(2):e178-83. doi: 10.1097/scs.0000000000005136

32. Yue Q, Yu Y, Shi Z, Wang Y, Zhu W, Du Z, et al. Prediction of BRAF mutation status of craniopharyngioma using magnetic resonance imaging features. J Neurosurg (2018) 129(1):27-34. doi: 10.3171/2017.4.Jns163113

33. Chen X, Tong Y, Shi Z, Chen H, Yang Z, Wang Y, et al. Noninvasive molecular diagnosis of craniopharyngioma with MRI-based radiomics approach. BMC Neurol (2019) 19(1):6. doi: 10.1186/s12883-018-1216-Z

34. Zhao W, Xu Y, Yang Z, Sun Y, Li C, Jin L, et al. Development and validation of a radiomics nomogram for identifying invasiveness of pulmonary adenocarcinomas appearing as subcentimeter ground-glass opacity nodules. Eur J Radiol (2019) 112:161-8. doi: 10.1016/j.ejrad.2019.01.021

35. Zheng J, Kong J, Wu S, Li Y, Cai J, Yu H, et al. Development of a noninvasive tool to preoperatively evaluate the muscular invasiveness of bladder cancer using a radiomics approach. Cancer (2019) 125(24):4388-98. doi: 10.1002/cncr.32490

36. Zhu Y, Man C, Gong L, Dong D, Yu X, Wang S, et al. A deep learning radiomics model for preoperative grading in meningioma. Eur J Radiol (2019) 116:128-34. doi: 10.1016/j.ejrad.2019.04.022

37. Zhao S, Su Y, Duan J, Qiu Q, Ge X, Wang A, et al. Radiomics signature extracted from diffusion-weighted magnetic resonance imaging predicts outcomes in osteosarcoma. J Bone Oncol (2019) 19:100263. doi: 10.1016/ j.jbo. 2019.100263

38. Stache C, Hölsken A, Fahlbusch R, Flitsch J, Schlaffer SM, Buchfelder M, et al. Tight junction protein claudin-1 is differentially expressed in craniopharyngioma subtypes and indicates invasive tumor growth. Neurooncology (2014) 16(2):256-64. doi: 10.1093/neuonc/not195

Conflict of Interest: The authors declare that the research was conducted in the absence of any commercial or financial relationships that could be construed as a potential conflict of interest.

Copyright (C) 2021 Ma, Kang, Qiao, Zhang, Chen, Li, Gao and Gui. This is an openaccess article distributed under the terms of the Creative Commons Attribution License (CC BY). The use, distribution or reproduction in other forums is permitted, provided the original author(s) and the copyright owner(s) are credited and that the original publication in this journal is cited, in accordance with accepted academic practice. No use, distribution or reproduction is permitted which does not comply with these terms. 